\title{
Clinical Implications of Second and Third Trimester Surveillance Ultrasounds of Growth- Restricted Fetuses
}

\author{
Amanda M. Craig, MD ${ }^{1}$ Karampreet Kaur, BA ${ }^{2}$ Sarah A. Heerboth, BA ${ }^{2}$ Heidi Chen, $\mathrm{PhD}^{3}$ \\ Chelsea J. Lauderdale, MPH, APRN ${ }^{4}$ Chevis Shannon, MBA, MPH, PhD ${ }^{4}$ Lisa C. Zuckerwise, MD 5
}

${ }^{1}$ Department of Obstetrics and Gynecology, Vanderbilt University Medical Center, Nashville, Tennessee

2 Vanderbilt University School of Medicine, Nashville, Tennessee

${ }^{3}$ Department of Biostatistics, Vanderbilt University Medical Center, Nashville, Tennessee

${ }^{4}$ Surgical Outcomes Center for Kids, Monroe Carell Junior Children's Hospital at Vanderbilt, Nashville, Tennessee

${ }^{5}$ Division of Maternal-Fetal Medicine, Department of Obstetrics and Gynecology, Vanderbilt University Medical Center, Nashville,

Tennessee

AJP Rep 2021;11:e132-e136.
Address for correspondence Amanda M. Craig, MD, Department of Obstetrics and Gynecology, Duke University Medical Center, 2608 Erwin Road, Durham, NC 27705 (e-mail: amanda.craig@duke.edu).

\begin{abstract}
\section{Keywords}

- fetal growth restriction

- ultrasound

- pregnancy

- antenatal corticosteroids

Objective We sought to investigate the positive predictive value of ultrasounddiagnosed fetal growth restriction (FGR) for estimating small for gestational age (SGA) at birth. Secondary objectives were to describe clinical interventions performed as a result of FGR diagnosis.

Study Design This was a retrospective cohort of pregnancies diagnosed with FGR over 3 years at a single institution. Maternal demographics, antenatal and delivery data, and neonatal data were collected. Descriptive statistics and linear regression were conducted.

Results We included 406 pregnancies with diagnosis of FGR in second or third trimester. Median birth weight percentile was 17 (interquartile range: 5-50) and only $35.0 \%$ of these fetuses were SGA at birth. The positive predictive value of a final growth ultrasound below the 10th percentile for SGA at birth was $56.9 \%$. Patients averaged eight additional growth ultrasounds following FGR diagnosis. One hundred and fourteen $(28.1 \%$ ) received antenatal steroids prior to delivery, and $100 \%$ of those delivered after more than 7 days following administration. There were 6 fetal deaths and 14 neonatal deaths.

Conclusion In the majority of cases, pregnancies diagnosed with FGR during screening ultrasounds resulted in normally grown neonates and term deliveries. These patients may be receiving unnecessary ultrasounds and premature courses of corticosteroids.
\end{abstract}

received

August 5, 2020

accepted after revision

October 8, 2021
Dol https://doi.org/ 10.1055/s-0041-1740170 ISSN 2157-6998.

\footnotetext{
(C) 2021. The Author(s).

This is an open access article published by Thieme under the terms of the Creative Commons Attribution-NonDerivative-NonCommercial-License, permitting copying and reproduction so long as the original work is given appropriate credit. Contents may not be used for commercial purposes, or adapted, remixed, transformed or built upon. (https://creativecommons.org/ licenses/by-nc-nd/4.0/)

Thieme Medical Publishers, Inc., 333 Seventh Avenue, 18th Floor, New York, NY 10001, USA
} 
Fetal growth restriction (FGR) is a clinically significant diagnosis that is commonly defined as an estimated fetal weight (EFW) below the 10th percentile for gestational age by the Society for Maternal-Fetal Medicine (SMFM). ${ }^{1}$ As such, $10 \%$ of pregnancies will carry out this diagnosis, which is associated with an increased risk of stillbirth, neonatal death, hypoxic ischemic encephalopathy, and cerebral palsy. ${ }^{2-5}$ According to one study, perinatal mortality rates are estimated at 5 per 1,000 births for fetuses diagnosed prenatally with FGR, with perinatal death occurring between $24^{6 / 7}$ and 35 weeks' gestation. ${ }^{6}$ Of growth-restricted fetuses who are small for gestational age (SGA) at birth, the neonatal mortality rate is 2.8 per 1,000 at term. The mortality rate approaches 60 per 1,000 for those delivered prematurely. ${ }^{7}$ Approximately $20 \%$ of FGR cases are attributed to chromosomal disorders and congenital malformations, contributing to the high morbidity and mortality associated with FGR. ${ }^{8,9}$ The current approach for the diagnosis and management of FGR is not standardized, making it challenging for providers to balance the risks of FGR with the risks of more frequent prenatal monitoring. Therefore, it is important to better understand the utility of screening growth ultrasounds in identifying sustained FGR, predicting the birth of an SGA neonate, and preventing fetal morbidity and mortality.

Current guidelines recommend screening for FGR using fundal height measurements as a proxy for estimated fetal size throughout pregnancy; however, the sensitivity of fundal height measurement is significantly reduced with common maternal conditions such as obesity or uterine fibroids. ${ }^{2,10}$ Ultrasound is useful to estimate fetal weight in cases where fundal height is suboptimal or abnormal, as well as in pregnancies at increased risk for FGR. The percent error between EFW by ultrasound and birth weight can be as high as $20 \%$, leading to both false positives and missed diagnoses. This error can be attributed to resolution of ultrasound images and the type and number of anatomic parameters measured. ${ }^{11}$ Over the past 10 years, the total number of ultrasound examinations performed during a pregnancy has continued to increase. ${ }^{12,13}$ One study found that the average number of ultrasounds per pregnancy across the United States has increased from 1.5 to 2.7 between 1995 and 2006. For high-risk pregnancies, the average number of ultrasounds increased from 2.2 to 4.2. ${ }^{12}$ Routine use of screening growth ultrasounds has been found to increase detection rates of FGR, which has been associated with increased rates of preterm induction of labor and cesarean deliveries, without decreasing the rate of adverse perinatal outcomes. ${ }^{14-16}$ We do acknowledge that there is currently no universally accepted growth curve for diagnosing FGR in the antenatal period which contributes to the variability in detection rates and establishing universal protocols for screening and management. This study sought to investigate the positive predictive value of ultrasound-diagnosed FGR in the second or third trimester for persistent growth restriction at birth. Our secondary objectives were to describe the clinical interventions performed as a result of FGR diagnosis, including antenatal corticosteroid administration and additional growth ultrasounds.

\section{Materials and Methods}

We conducted a retrospective review of all pregnancies diagnosed with FGR by the Division of Maternal-Fetal Medicine at Vanderbilt University Medical Center from January 1, 2014, to December 31, 2016. The Vanderbilt Institutional Review Board approved this study on February 10, 2017 (IRB \#170218). Pregnancies with the ultrasound finding of EFW below the 10th percentile for gestational age were identified within our ultrasound database, and those with first or second trimester ultrasound confirming accurate dating were included. EFW percentile was calculated using the Hadlock formula. Pregnancies with known fetal structural or chromosomal abnormalities were excluded. Fenton growth curves were used for preterm infants and the World Health Organization growth curves were used for term infants to define SGA.

During the time period of this study, it was our practice to obtain growth ultrasounds at 4-week intervals for patients with medical comorbidities complicating their pregnancy that put them at risk for pregnancies complicated by FGR, or in whom fundal height measurement was suboptimal due to maternal obesity or uterine anomalies. Following FGR diagnosis, we increased the frequency of growth assessment to 3-week intervals, initiated twice weekly antenatal surveillance with biophysical profile (BPP) or fetal nonstress test (NST), and obtained weekly umbilical artery Doppler assessment.

Our primary outcome was percentage of FGR pregnancies that resulted in a SGA neonate. Diagnosis of SGA was made utilizing the birth weight recorded for the neonate in the electronic medical record (EMR). Maternal data, including maternal demographics and comorbidities, were also collected from the EMR. We additionally collected data on antenatal corticosteroid administration, BPP scores, fetal NST results, and umbilical artery Doppler assessments in patients who received such interventions or surveillance procedures. All data were stored in a secure REDCap database. ${ }^{17}$ Descriptive statistics were conducted using the Kruskal-Wallis' test and Spearman's rank correlation. Statistical significance was set a priori at $p<0.05$.

\section{Results}

\section{Growth Restriction throughout Pregnancy}

We identified 406 cases of FGR during our study period that met inclusion criteria. Thirty-seven $(9.1 \%)$ were twin pregnancies. Twin pregnancies were treated as individual fetuses with regard to data analysis. Baseline maternal characteristics are shown in - Table $\mathbf{1}$. The most common maternal medical comorbidities were obesity (44\%), maternal tobacco use (19\%), congenital infection (11\%), and hypertension (10\%). Maternal medical comorbidities were absent in 35\% of our population. The median gestational age at diagnosis of FGR was 25 weeks (interquartile range [IQR]: 20-31). The median EFW percentile at diagnosis was 7 (IQR: 5-8). The median birth weight percentile of our cohort was 17 (IQR: 5-50). 
Table 1 Maternal demographic data and comorbidities $(N=406)$

\begin{tabular}{|c|c|}
\hline Age at EDD, mean $\pm S D$ & $28.69 \pm 6.83$ \\
\hline Gravidity, mean $\pm S D$ & $2 \pm 1.6$ \\
\hline Parity, mean $\pm S D$ & $1 \pm 1.2$ \\
\hline $\mathrm{BMI}$, mean $\pm \mathrm{SD}$ & $29.8 \pm 7.67$ \\
\hline \multicolumn{2}{|l|}{ Race, $n(\%)$} \\
\hline Caucasian & $258(63.5 \%)$ \\
\hline African American & $68(16.7 \%)$ \\
\hline Asian & $33(8.1 \%)$ \\
\hline Other & $47(11.7 \%)$ \\
\hline \multicolumn{2}{|l|}{ Maternal comorbidities } \\
\hline None & $143(35.2 \%)$ \\
\hline Obesity & $178(43.8 \%)$ \\
\hline Tobacco use & 75 (18.5\%) \\
\hline Congenital infection & $46(11.3 \%)$ \\
\hline Hypertension & $41(10.1 \%)$ \\
\hline Asthma & $38(9.4 \%)$ \\
\hline Hypothyroidism & $31(7.6 \%)$ \\
\hline Diabetes & $15(3.7 \%)$ \\
\hline Cardiac disease & $13(3.2 \%)$ \\
\hline Uterine anomalies & $13(3.2 \%)$ \\
\hline Seizure disorder & $12(3.0 \%)$ \\
\hline Fibroids & $11(2.7 \%)$ \\
\hline Hyperthyroidism & $6(1.5 \%)$ \\
\hline Lupus & $3(0.7 \%)$ \\
\hline
\end{tabular}

Abbreviations: BMI, body mass index; EDD, estimated delivery date; SD, standard deviation.

Within our cohort of pregnancies complicated by FGR, 65\% (264) of neonates were normally grown at birth or above the sex-specific 10th percentile for birth weight. Fifty-one percent (209) of our cohort had EFWs below the 10th percentile at the final documented growth ultrasound prior to delivery, and of those pregnancies, 119 were born SGA. We calculated that the positive predictive value of a final growth ultrasound EFW below the 10th percentile for predicting SGA at birth was $56.9 \%$. The mean EFW percentile at final growth ultrasound for fetuses that were FGR at final ultrasound and in fact SGA at birth was $4.3 \pm 1.8$, which was significantly lower $(p<0.001)$ than the mean EFW percentile for fetuses that were FGR at final ultrasound and not SGA at birth $(6.2 \pm 2.3)$.

\section{Clinical Interventions}

The median total number of ultrasound examinations, including studies for growth, BPP, and Doppler assessment, performed during each FGR pregnancy was 8 (IQR: 6-11). Most patients had two (20\%), three (27\%), or four (23\%) growth ultrasounds during their pregnancy. We performed five or more growth ultrasounds in 77 patients (19\%). Final growth ultrasound occurred at a median gestational age of 35 weeks (IQR: 33-37). Regression analysis found no statistically significant relationship between the gestational age at delivery and total number of growth ultrasounds.

In our cohort, 114 patients (28\%) received a course of antenatal corticosteroids, with $96 \%$ completing the two-dose course prior to delivery. All of these patients remained pregnant more than 7 days after administration of steroid course. Among patients who received antenatal corticosteroids, $21(15 \%)$ received a second "rescue course" of antenatal corticosteroids. The median gestational age for the initial course of steroids was 30 weeks (IQR: 26-32) with a median of 21 days (IQR: 3-47) between the first day of receiving betamethasone and date of delivery. Six patients (27\%) of those who received a "rescue course" remained pregnant more than 7 days after the initiation of the steroid course, without indication for delivery or onset of labor. The median interval between rescue course and delivery was 4 days (IQR: 1-15). Median gestational age at delivery for all patients who received antenatal corticosteroids was 34 weeks (IQR: 32-37). Of the 114 patients who received antenatal steroids, $84(74 \%)$ delivered prior to 37 weeks' gestation.

\section{Delivery and Neonatal Outcomes}

The median gestational age at delivery among our cohort was 38 weeks (IQR: 36-39). Thirty-two percent (130) patients delivered preterm. Twenty-two percent of pregnancies delivered in the setting of spontaneous labor. Indications for delivery were characterized as fetal in $48 \%$ and maternal in $30 \%$ of pregnancies (-Table 2 ). The most common indications for delivery, excluding the population that went into spontaneous labor, included scheduled induction of labor (30\%), scheduled repeat cesarean section (17\%),

Table 2 Indications for delivery, excluding spontaneous labor $(N=316)$

\begin{tabular}{|l|l|}
\hline Maternal indications & $\boldsymbol{n}(\%)$ \\
\hline Scheduled repeat cesarean section & $53(16.8 \%)$ \\
\hline Preeclampsia or gestational hypertension & $44(13.9 \%)$ \\
\hline $\begin{array}{l}\text { Medical comorbidities (e.g., lupus, sickle cell } \\
\text { disease, chronic hypertension) }\end{array}$ & $16(5.1 \%)$ \\
\hline Poorly controlled diabetes mellitus & $5(1.6 \%)$ \\
\hline Placental abnormalities & $3(0.9 \%)$ \\
\hline Fetal indications & $93(29.4 \%)$ \\
\hline Scheduled induction of labor & $30(9.5 \%)$ \\
\hline Abnormal growth interval & $22(7.0 \%)$ \\
\hline Oligohydramnios & $19(6.0 \%)$ \\
\hline Abnormal nonstress test & $18(5.7 \%)$ \\
\hline $\begin{array}{c}\text { Abnormal umbilical artery Doppler } \\
\text { ultrasounds }\end{array}$ & $8(2.5 \%)$ \\
\hline Abnormal biophysical profile & $4(1.3 \%)$ \\
\hline Fetal demise & $1(0.3 \%)$ \\
\hline Decreased fetal movement at term &
\end{tabular}


preeclampsia or gestational hypertension (14\%), and abnormal growth interval (10\%). There were six cases (1.5\%) of fetal demise in our study population. Median gestational age at fetal demise was 31 weeks (IQR: 27-33.5). All six pregnancies were complicated by maternal obesity. One pregnancy had multiple gestations. Four of these patients were prescribed a steroid course. Five of the fetal demises (83.3\%) were SGA at delivery.

There were 14 cases $(3.4 \%)$ of neonatal demise in our study population. The median gestational age at delivery for these neonates was 33.5 weeks (IQR: 26.8-35), and 9 (64.3\%) were SGA at birth. The most common neonatal complications among all pregnancies were neonatal intensive care unit admission (34\%), hyperbilirubinemia (27\%), hypothermia (12\%), and respiratory distress syndrome (10\%). As expected, all neonatal complications were significantly more likely in cases of earlier gestational age at delivery $(p<0.001)$.

\section{Discussion}

Our study highlights the diagnostic challenges and clinical implications associated with diagnosing FGR during screening growth ultrasounds. In our study, patients had a median of eight ultrasound examinations, which is twice the estimated average number of ultrasounds per high-risk pregnancy in the United States. ${ }^{12}$ This is likely because following FGR diagnosis, patients underwent twice weekly fetal surveillance, including at least weekly ultrasounds for BPP and Doppler assessment, in adherence with established clinical guidelines. ${ }^{1}$ Interestingly, the majority of fetuses diagnosed with FGR were normally grown at birth, with only 35\% delivering SGA. Similar findings were published from a study conducted in France, which found that $21.7 \%$ of SGA neonates were diagnosed of FGR during pregnancy. ${ }^{14}$ Additionally, Sovio et al found that the positive predictive value for SGA after an ultrasound diagnosis of FGR is estimated to be only approximately $50 \%{ }^{15}$ Our results support these prior publications as we calculated a positive predictive value of $56.9 \%$, which further contributes to the available evidence that screening growth ultrasounds may be relatively imprecise tools for predicting underweight neonates. We did find that of the fetuses that were FGR at final growth ultrasound, the ones who were SGA had an EFW below the fifth percentile, while the ones who were not SGA had an EFW above the fifth percentile. Studies have found that EFW below fifth percentile has higher morbidity and a stillbirth as high as $2.5 \%$. 2,18

The American College of Obstetricians and Gynecologists recommends corticosteroid administration when delivery is imminent and gestational age is between $24^{0 / 7}$ and $36^{6 / 7}$ weeks to reduce the risk of respiratory distress syndrome, intracranial hemorrhage, necrotizing enterocolitis, and perinatal death. ${ }^{19}$ SMFM recommends antenatal corticosteroids if delivery is anticipated before $33^{6 / 7}$ weeks' gestation or for pregnancies between $34^{0 / 7}$ and $36^{6 / 7}$ weeks that are at risk of preterm delivery within 7 days. ${ }^{1}$ Maximum benefit has been shown for neonates in whom delivery occurs 2 to 7 days following steroid administration. ${ }^{20,21}$ The median gestation- al age for the initial course of steroids was 30 weeks (IQR: 26-32) in our population. Our results were consistent with prior studies that have reported a high incidence $(55-80 \%)$ of premature steroid dosing in patients at risk for preterm delivery. $^{22,23}$ We acknowledge that our data collection spanned the time during which the Antenatal Late Preterm Steroids trial was published and those results may have impacted our clinical practice for fetuses between $34^{0 / 7}$ and $36^{6 / 7}$ weeks. ${ }^{24}$

Prior studies have found that FGR is an important risk factor for unexplained intrauterine death. One study found $52 \%$ of unexplained stillbirths were growth restricted. ${ }^{25}$ Another high-powered study found a perinatal mortality rate of 5.4/1,000 births among pregnancies complicated by FGR. ${ }^{6}$ In our study, $1.5 \%$ of pregnancies were complicated by fetal demise. Five of the six demised pregnancies remained growth restricted at the time of death. SMFM recommends delivery at 38 to 39 weeks of gestation with FGR and normal umbilical artery Doppler ultrasound. ${ }^{1}$ The median gestational age at delivery in our population was 38 weeks (IQR: 36-39), which remained the same when 18 fetuses with the indication of abnormal umbilical artery Dopplers for delivery were removed from analysis.

Our results and conclusions are limited by the retrospective nature of our study. We cannot directly compare methods of screening for and surveillance of FGR at our institution with alternative practices. We additionally were not able to compare outcomes of our FGR population to a nongrowthrestricted population. Despite these limitations, this study investigated the impact of an FGR diagnosis on a large cohort of more than 400 pregnancies with a similar incidence of fetal and neonatal demise to the national perinatal mortality rate for pregnancies affected by FGR. ${ }^{26}$

\section{Conclusion}

Overall, we found a majority of our pregnancies complicated by the diagnosis of FGR during routine second and third trimester screening ultimately had normally grown neonates, with birth weights above the 10th percentile. The diagnosis of FGR potentially led to excess ultrasound examinations, premature corticosteroid administration, and likely increased health care costs. Future studies are needed to compare different methods of screening for abnormal fetal growth, and to determine the optimal cutoff for diagnosing FGR that balances the goals of avoiding overtreatment with reducing the incidence of perinatal and neonatal morbidity and mortality.

\section{Conflict of Interest \\ None declared.}

\section{Acknowledgments}

The authors thank Edwina McNeill-Simaan, research coordinator in the Vanderbilt Department of Obstetrics and Gynecology, for her assistance in preparing the IRB submission and in creation of the RedCap database. 


\section{References}

1 Society for Maternal-Fetal Medicine(SMFM) Martins JG, Biggio JR, Abuhamad A. Society for Maternal-Fetal Medicine Consult Series \#52: diagnosis and management of fetal growth restriction: (Replaces Clinical Guideline Number 3, April 2012). Am J Obstet Gynecol 2020;223(04):B2-B17

2 ACOG practice bulletin no. 134: fetal growth restriction. Obstet Gynecol 2013;121(05):1122-1133

3 Gardosi J, Madurasinghe V, Williams M, Malik A, Francis A. Maternal and fetal risk factors for stillbirth: population based study. BMJ 2013;346:f108

4 Chauhan SP, Beydoun H, Chang E, et al. Prenatal detection of fetal growth restriction in newborns classified as small for gestational age: correlates and risk of neonatal morbidity. Am J Perinatol 2014;31(03):187-194

5 Mendez-Figueroa H, Truong VT, Pedroza C, Chauhan SP. Morbidity and mortality in small-for-gestational-age infants: a secondary analysis of nine MFMU Network studies. Am J Perinatol 2017;34 (04):323-332

6 Unterscheider J, O'Donoghue K, Daly S, et al. Fetal growth restriction and the risk of perinatal mortality-case studies from the multicentre PORTO study. BMC Pregnancy Childbirth 2014;14:63

7 Ray JG, Park AL, Fell DB. Mortality in infants affected by preterm birth and severe small-for-gestational age birth weight. Pediatrics 2017;140(06):e20171881

8 Bamfo JE, Odibo AO. Diagnosis and management of fetal growth restriction. J Pregnancy 2011;2011:640715

9 Resnik R. Intrauterine growth restriction. Obstet Gynecol 2002;99 (03):490-496

10 Goetzinger KR, Tuuli MG, Odibo AO, Roehl KA, Macones GA, Cahill AG. Screening for fetal growth disorders by clinical exam in the era of obesity. J Perinatol 2013;33(05):352-357

11 Committee on Practice Bulletins-Obstetrics and the American Institute of Ultrasound in Medicine. 175: Ultrasound in pregnancy. Obstet Gynecol 2016;128(06):e241-e256

12 Siddique J, Lauderdale DS, VanderWeele TJ, Lantos JD. Trends in prenatal ultrasound use in the United States: 1995 to 2006. Med Care 2009;47(11):1129-1135

13 O'Keeffe DF, Abuhamad A. Obstetric ultrasound utilization in the United States: data from various health plans. Semin Perinatol 2013;37(05):292-294

14 Monier I, Blondel B, Ego A, Kaminiski M, Goffinet F, Zeitlin J. Poor effectiveness of antenatal detection of fetal growth restriction and consequences for obstetric management and neonatal outcomes: a French national study. BJOG 2015;122(04):518-527
15 Sovio U, White IR, Dacey A, Pasupathy D, Smith GCS. Screening for fetal growth restriction with universal third trimester ultrasonography in nulliparous women in the Pregnancy Outcome Prediction (POP) study: a prospective cohort study. Lancet 2015;386(10008):2089-2097

16 Gabbay-Benziv R, Aviram A, Hadar E, et al. Pregnancy outcome after false diagnosis of fetal growth restriction. J Matern Fetal Neonatal Med 2017;30(16):1916-1919

17 Harris PA, Taylor R, Thielke R, Payne J, Gonzalez N, Conde JG. Research Electronic Data Capture (REDCap)-a metadata-driven methodology and workflow process for providing translational research informatics support. J Biomed Inform 2009;42(02): 377-381

18 Minior VK, Divon MY. Fetal growth restriction at term: myth or reality? Obstet Gynecol 1998;92(01):57-60

19 Committee on Obstetric Practice. Committee Opinion No. 713: antenatal corticosteroid therapy for fetal maturation. Obstet Gynecol 2017;130(02):e102-e109

20 Romagnoli S, Zagli G, Tuccinardi G, et al. Postoperative acute kidney injury in high-risk patients undergoing major abdominal surgery. J Crit Care 2016;35:120-125

21 Roberts D, Brown J, Medley N, Dalziel SR. Antenatal corticosteroids for accelerating fetal lung maturation for women at risk of preterm birth. Cochrane Database Syst Rev 2017;3: CD004454

22 Boesveld M, Heida KY, Oudijk MA, Brouwers HA, Koenen SV, Kwee A. Evaluation of antenatal corticosteroid prescribing patterns among 984 women at risk for preterm delivery. J Matern Fetal Neonatal Med 2014;27(05):516-519

23 Adams TM, Kinzler WL, Chavez MR, Vintzileos AM. The timing of administration of antenatal corticosteroids in women with indicated preterm birth. Am J Obstet Gynecol 2015;212(05):645. e1-645.e4

24 Gyamfi-Bannerman C, Thom EA, Blackwell SC, et al; NICHD Maternal-Fetal Medicine Units Network. Antenatal betamethasone for women at risk for late preterm delivery. $\mathrm{N}$ Engl J Med 2016;374(14):1311-1320

25 Frøen JF, Gardosi JO, Thurmann A, Francis A, Stray-Pedersen B. Restricted fetal growth in sudden intrauterine unexplained death. Acta Obstet Gynecol Scand 2004;83(09):801-807

26 Lees C, Marlow N, Arabin B, et al; TRUFFLE Group. Perinatal morbidity and mortality in early-onset fetal growth restriction: cohort outcomes of the trial of randomized umbilical and fetal flow in Europe (TRUFFLE). Ultrasound Obstet Gynecol 2013;42 (04):400-408 